\title{
Stochastic Linear Quadratic Optimal Control for Discrete-time Systems with Inequality Constraint and Markovian Jumps
}

\author{
Wenying WANG**1, Zhiming ZHANG ${ }^{2}$, Running MIAO ${ }^{1}$ \\ ${ }^{1}$ College of Mathematics and System Science, Shandong University of Science and Technology \\ Qingdao 266590, Shandong, People's Republic of China. \\ ${ }^{2}$ College of Economics and Management, Shandong University of Science and Technology \\ Qingdao 266590, Shandong, People's Republic of China
}

*Corresponding author. Email: wenyingwang1990@163.com

Keywords: Linear quadratic optimal control; Discrete-time system; Indefinite control weights; Markovian jumps

\begin{abstract}
This paper primarily discusses the linear quadratic optimal control problem for discrete-time stochastic sys- tems with indefinite control weights and constraint and Markovian jumps. We use the Karush-Kuhn-tucker (KKT) theorem basically in this paper. It is testified that the well- posedness and the attainability are equivalent about the stochastic linear quadratic optimal control problem with Markovian jumps. Furthermore, the solution of the generalized difference Riccati equation (GDRE) can indicate an optimal control.
\end{abstract}

\section{Introduction}

In this paper, we focus our attention on the finite horizon indefinite stochastic linear quadratic control with Markovian jumps and terminal inequality constraint. There are homologous constraints in $H_{\infty}$ filtering problems [1,2]. The summary of this paper is arranged as follows. In Section 2, we render some definitions and preliminaries. Section 3 elaborates and proves our primary theorems. Here a necessary condition about the existence of linear optimal state feedback control with Markovian jumps is obtained. Furthermore, it is illustrated that the solvability of the GDRE, the attainability and the well-posedness of the linear quadratic problem are all equivalent.

We shall find that it is convenient to apply the following marks in this paper. $\operatorname{tr}(A)$ indicates

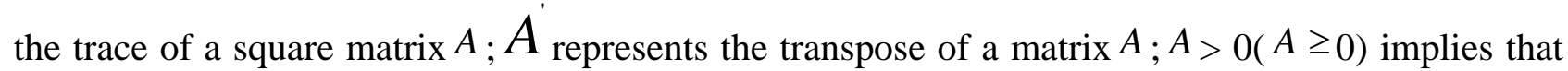
$\mathrm{A}$ is positive definite (positive semi-definite) symmetric matrix; $\mathrm{E}[\mathrm{x}]$ signifies the mathematical expectation of a arbitrary variable $x ; A^{+}$represents the Moore-Penrose generalized inverse matrix of a matrix $A ; R^{l}$ is the ${ }^{l}$-dimensional Eulerian space with the usual 2-norm \|\|$; R^{m \times n}$ means the vector space of all $m \times n$ matrices with entries in $R$; $I$ represents the identity matrix with suitable dimension; $N_{t}=\{0,1,2 \cdots, t-1\}$ and $\bar{N}=\{1, \cdots, N\}$.

\section{Definitions and preliminaries}

Given a probability space $(\Omega, F, P)$, think about the discrete-time stochastic system with Markovian jumps:

$$
\left\{\begin{array}{l}
x(l+1)=A_{\theta(l)}(l) x(l)+B_{\theta(l)}(l) u(l)+C_{\theta(l)}(l) x(l) \omega^{x}(l)+D_{\theta(l)}(l) u(l) \omega^{u}(l)+\omega(l), l \in N_{T} \\
b_{i 1} X_{1}(T)+b_{i 2} X_{2}(T)+\cdots+b_{i n} X_{n}(T) \leq \xi_{i}
\end{array}\right.
$$


where $x(0)=x_{0} \in R^{n}$ is the given initial state, $x(l) \in R^{n}$ and $u(l) \in R^{m}$ are, severally, the system state and controlled input of the system, $\theta(l)$ is a time-varying Markov chain with the transition probability

matrix

$\mathrm{P}(l)=\left(p_{i j}(l)\right), p_{i j}(l)=P(\theta(l+1)=j \mid \theta(l)=i) \cdot A_{\theta(l)}(l), C_{\theta(l)}(l) \in R^{n}, B_{\theta(l)}(l), D_{\theta(l)}(l) \in R^{n \times m}$ are matrix valued functions of appropriate dimensions. Without loss of generality, we suppose that $\omega^{x}(l), \quad \omega^{u}(l)$ are scalar arbitrary variables and independent of the Markov chain $\theta(\cdot)$. The initial value $\theta(0)=\theta_{0}$ is also independent of the noise $\omega(l)\left(l \in N_{T}\right)$. In addition, $\omega^{x}(l), \omega^{u}(l), \omega(l)$ satisfy the following

$$
E\left[\omega^{x}(l)\right]=E\left[\omega^{u}(l)\right]=E[\omega(l)]=0, E\left[\left(\omega^{x}(l)\right)^{2}\right]=E\left[\left(\omega^{u}(l)\right)^{2}\right]=1, E\left[\omega(l) \omega^{\prime}(l)\right]=V(l),
$$

$E\left[\omega^{x}(l) \omega^{u}(l)\right]=\rho^{x u}(l), E\left[\omega(l) \omega^{x}(l)\right]=E\left[\omega(l) \omega^{u}(l)\right]=0$.

We indicate $F_{l}$ the $\sigma-$ algebra generated by $\left(\omega^{x}(l), \omega^{u}(l), \omega(l)\right)$, that is to say, $\quad F_{l}=\left\{\sigma\left(\omega^{x}(l), \quad \omega^{u}(l), \omega(l)\right): l \in N_{T}\right\} \cdot u(\cdot)$ is part of the admissible control set $U_{a d}=\left\{u(l) \in R^{m}: E\left[\sum_{l=0}^{T-1}|u(l)|^{2}<+\infty\right]\right\} \quad \xi_{i} F_{T}$ measurable square integrable stochastic process, that is $E\left[\left|\xi_{i}\right|\right]<+\infty$. ${ }_{\text {Let }} N_{r \times n}=\left(b_{i j}\right), \xi=\left(\xi_{r \times n}, \xi_{2}, \cdots, \xi_{r}\right)$, afterwards the constraint in (2.1) can be indicated as $N x(T) \leq \xi$, where $b_{i j}$ is constant. $N$ has row full rank.

To make the expressions tighter, we apply some signs that will be used later. Let $P$ indicates a collection of symmetric matrices which includes the time $l$ and the mode of operation $i$. that is, $P=\left\{P_{\theta(l)}(l) \in S^{n}: l \in \quad N_{T+1}, \theta(l) \in \bar{N}\right\}, \quad P(l)=\left[P_{1}(l), P_{2}(l), \cdots, P_{N}(l)\right] . \quad$ For $\quad P \quad$ and $i \in \bar{N}_{\text {set }} \psi_{i}(l, P(l+1))=\sum_{j=1}^{N} p_{i j}(l) P_{j}(l+1)$.

We deem the following cost function connected with the system (2.1).

$J\left(X_{0}, u(0), \cdots, u(T-1)\right)=\sum_{l=0}^{T-1} E\left[\left(x^{\prime}(l) Q_{\theta(l)}(l) x(l)+u^{\prime}(l) R_{\theta(l)}(l) u(l)\right)+x^{\prime}(T) S_{\theta(T)} x(T)\right]$,

where $Q_{\theta(l)}(l), S_{\theta(T)}$ and $R_{\theta(l)}(l)$ are symmetric matrices with suitable dimension, which are possible indefinite.

Defining as below

$$
V\left(X_{0}\right)=\inf _{u(0), \cdots, u(T-1)} J\left(X_{0}, u(0), \cdots, u(T-1)\right) .
$$

In the continuation, we study the linear quadratic problem for the systems (2.1)-(2.3), in other words, seeking a control to minimize $J\left(X_{0}, u(0), \cdots, u(T-1)\right)$. First of all, we particularize some helpful definitions and lemmas that are indispensable to research our main results.

Definition 2.1 If $V\left(X_{0}\right)>-\infty$ for any $X_{0}$, systems (2.1)-(2.3) are called well-posed.

Definition 2.2 If there exists an admissible control $\boldsymbol{u}_{*}(0), \cdots, \boldsymbol{u}_{*}(T-1)$ such that $V\left(\boldsymbol{X}_{0}\right)=J\left(\boldsymbol{X}_{0}, \boldsymbol{U}_{*}(0), \cdots, \boldsymbol{U}_{*}(T-1)\right)$. Then systems (2.1)-(2.3) are deemed to be attainable, $\left(\boldsymbol{u}_{*}(0), \cdots, \boldsymbol{u}_{*}(T-1)\right)$ is called an optimal control. 
If a linear feedback control is optimal for the linear quadratic problem (2.1)-(2.3), like that it must be optimal linear feedback control of the listed below form $u(l)=K_{\theta(l)}(l) x(l), l \in N_{T}$, where $K_{\theta(l)}^{(l)}$ is matrix-valued function.

MP(mathematical programming)

$$
\begin{gathered}
\min f(x) \\
\text { s.t. }\left\{\begin{array}{l}
g(x) \leq 0 \\
h(x)=0
\end{array}\right. \\
\text { where } g(x)=\left(g_{1}(x), \cdots, g_{p}(x)\right), h(x)=\left(h_{1}(x), \cdots, h_{q}(x)\right) .
\end{gathered}
$$

There are two definitions, i.e. Regularity condition and Regular point, in [3].

Lemma 2. 3 (KKT Theorem)[3] In MP above, suppose that the objective function $\mathrm{f}$ and the constraint functions $g=\left(g_{1}, \cdots, g_{p}\right)^{\prime}, h=\left(h_{1}, \cdots, h_{q}\right)^{\prime}$ are continuously differentiable at a point $X^{*}$. If $X^{*}$ is a local minimum that satisfies some regularity conditions, then there exist a vector $\lambda \geq 0$ in $R^{m}$ and a vector $\mu$ in $R^{n}$,called KKT multipliers, such that

$$
\left\{\begin{array}{l}
\nabla_{x} L\left(x^{*}, \lambda^{*}, \mu^{*}\right)=0 \\
\lambda^{\prime} g\left(x^{*}\right)=0
\end{array}\right.
$$

where the Lagrangian function $L(x, \lambda, \mu)=f(x)+\lambda^{\prime} g+\mu h(x)$.

\section{Main results under state feedback control}

Theorem 3.1 If the linear quadratic optimal control problem (2.1)-(2.3) is attainable on the basis of $u(l)=K_{\theta(l)}(l) x(l)$.At the same time, the regular point $\left(u_{*}(l), X_{*}(l)\right)$ is a veritable optimal solution of problem (2.1)-(2.3), afterwards there are solutions $(P(l), \mu)$ with $0 \leq \mu \in R^{1}, l \in N_{T}$ on the following GDRE

$$
\begin{aligned}
& P_{i}(l)=A_{i}^{\prime}(l) \psi_{i}(l, P(l+1)) A_{i}(l)+C_{i}^{\prime}(l) \psi_{i}(l, P(l+1)) C_{i}(l)+Q_{i}(l)-H_{i}^{\prime}(l) G_{i}^{\prime}(l) H_{i}(l) \\
& H_{i}(l)=B_{i}^{\prime}(l) \psi_{i}(l, P(l+1)) A_{i}(l)+\rho^{x u} D_{i}(l) \psi_{i}(l, P(l+1)) C_{i}(l) \\
& G_{i}(l)=R_{i}(l)+B_{i}^{\prime}(l) \psi_{i}^{(l, P(l+1))} B_{i}(l)+D_{i}^{\prime}(l) \psi_{i}(l, P(l+1)) D_{i}(l) \geq 0, l \in N_{T} \\
& \mu=\frac{\operatorname{tr}\left[P_{\theta_{T}}(T)-S\right]}{\operatorname{tr}\left(N^{\prime} N\right)} \\
& \mu\left[\operatorname{tr}\left(X(T) N^{\prime} N\right)-\operatorname{tr}(M)\right]=0, M=E[\xi \xi] .
\end{aligned}
$$

Moreover,

$$
u_{*}(l)=\left[-G_{\theta(l)}^{+}(l) H_{\theta(l)}(l)+Y_{\theta(l)}(l)-G_{\theta(l)}^{\dot{*}}(l) G_{\theta(l)}(l) Y_{\theta(l)}(l)\right] x(l), Y_{\theta(l)}(l) \in R^{m \times n}, l \in N_{T} .
$$

along with $V_{i}\left(X_{0}\right)=\sum_{l=0}^{T-1} \operatorname{tr}\left[V_{i}(l) \psi_{i}(l, P(l+1))\right]+X_{0} P_{\theta_{0}}(0) X_{0}-\mu \operatorname{tr}(M)$.

Proof We know $X(l)=E\left[x(l) x^{\prime}(l)\right]$ and $u(l)=K_{\theta(l)}(l) x(l)$ with any $l \in N_{T}$. The linear quadratic problem (2.1)-(2.3) can be replaced by the following optimization, which is can be proved. 


$$
\begin{aligned}
& \min _{K(0), \cdots, K(T-1)} \sum_{l=0}^{T-1} \operatorname{tr}\left[\left(Q_{\theta(l)}(l)+K_{\theta(l)}^{\prime}(l) R_{\theta(l)}(l) K_{\theta(l)}(l)\right) X(l)\right]+\operatorname{tr}[S X(T)] \\
& \left(X(l+1)=\sum_{j=1}^{N} p_{i j}(l)\left[A_{\theta(l)}(l) X(l) A_{\theta(l)}^{\prime}(l)+C_{\theta(l)}(l) X(l) C_{\theta(l)}^{\prime}(l)+B_{\theta(l)}(l) K_{\theta(l)}(l) X(l) K_{\theta(l)}^{\prime}(l) B_{\theta(l)}^{\prime}(l)\right.\right. \\
& +D_{\theta(l)}(l) K_{\theta(l)}(l) X(l) K_{\theta(l)}^{\prime}(l) D_{\theta(l)}^{\prime}(l)+A_{\theta(l)}(l) X(l) K_{\theta(l)}^{\prime}(l) B_{\theta(l)}^{\prime}(l)+B_{\theta(l)}(l) K_{\theta(l)}(l) X(l) A_{\theta(l)}^{\prime}(l) \\
& \left\{+\rho^{x u} D_{\theta(l)}^{(l)} K_{\theta(l)}(l) X(l) C_{\theta(l)}^{\prime}(l)+\rho^{x u} C_{\theta(l)}(l) X(l) K_{\theta(l)}^{\prime}(l) D_{\theta(l)}^{\prime}(l)\right]+V_{\theta(l)}(l) \\
& X(0)=E\left[x(0) X^{\prime}(0)\right]=X_{0} X_{0}^{\prime} \\
& \operatorname{tr}\left[X(T) N^{\prime} N\right] \leq \operatorname{tr} M, M=E[\xi \xi]
\end{aligned}
$$

Apparently, the problem (3.2) is a MP problem as follows

$$
\min f\left[X(l), K_{\theta(l)}(l)\right]
$$

s.t. $\left\{\begin{array}{l}h\left[X(l), K_{\theta(l)}(l)\right]=0 \\ g[X(T)] \leq 0, l \in N_{T}\end{array}\right.$

where

$$
\begin{aligned}
& f\left[X(l), K_{\theta(l)}(l)\right]=\sum_{l=0}^{T-1} \operatorname{tr}\left[\left(Q_{\theta(l)}(l)+K_{\theta(l)}^{\prime}(l) R_{\theta(l)}(l) K_{\theta(l)}(l)\right) X(l)\right]+\operatorname{tr}[S X(T)] \\
& h\left[X(l), K_{\theta(l)}(l)\right]=\sum_{j=1}^{N} p_{i j}(l)\left[A_{\theta(l)}(l) X(l) A_{\theta(l)}^{\prime}(l)+C_{\theta(l)}(l) X(l) C_{\theta(l)}^{\prime}(l)+B_{\theta(l)}(l) K_{\theta(l)}(l) X(l) K_{\theta(l)}^{\prime}(l) B_{\theta(l)}^{\prime}(l)\right. \\
& +D_{\theta(l)}(l) K_{\theta(l)}(l) X(l) K_{\theta(l)}^{\prime}(l) D_{\theta(l)}^{\prime}(l)+A_{\theta(l)}(l) X(l) K_{\theta(l)}^{\prime}(l) B_{\theta(l)}^{\prime}(l)+B_{\theta(l)}(l) K_{\theta(l)}(l) X(l) A_{\theta(l)}^{\prime}(l) \\
& \left.+\rho^{X u} D_{\theta(l)}(l) K_{\theta(l)}(l) X(l) C_{\theta(l)}^{\prime}(l)+\rho^{X u} C_{\theta(l)}(l) X(l) K_{\theta(l)}^{\prime}(l) D_{\theta(l)}^{\prime}(l)\right]+V_{\theta(l)}(l) \\
& g[X(T)]=\operatorname{tr}\left[X(T) N^{\prime} N\right]-M .
\end{aligned}
$$

By means of KKT Theorem, the Lagrangian function is defined as following

$$
L_{i}(X(l), K(l), P(l+1), \mu)=f\left[X(l), K_{\theta(l)}(l)\right]+\sum_{l=0}^{T-1} \operatorname{tr}\left\{P_{j}(l+1)\left[h\left[X(l), K_{\theta(l)}(l)\right]-X(l+1)\right]\right\}+\mu g[X(T)]
$$

where the matrices $P(0), P(1), \cdots, P(T)$ are Lagrangian multipliers and $\mu \in R^{1}$.

Furthermore, the above result is apparent.

$$
\frac{\partial L}{\partial X(l)}=\frac{\partial L}{\partial K_{\theta(l)}(l)}=\frac{\partial L}{\partial X(T)}=0
$$

By means of caculating, we know that $P(l)$ and $\lambda$ satisfy the equation as follows

$$
\left[R_{i}(l)+B_{i}^{\prime}(l) \psi_{i}(l, P(l+1)) B_{i}(l)+D_{i}^{\prime}(l) \psi_{i}(l, P(l+1)) D_{i}(l)\right] K_{i}(l)
$$

$$
=-B_{i}^{\prime}(l) \psi_{i}(l, P(l+1)) A_{i}(l)-\rho^{x u} D_{i}(l) \psi_{i}(l, P(l+1)) C_{i}(l),
$$

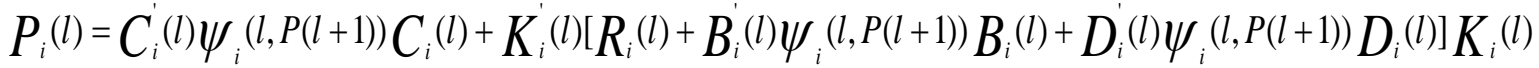

$+\left[A_{i}^{\prime}(l) \psi_{i}(l, P(l+1)) B_{i}(l)+\rho^{x u} D_{i}^{\prime}(l) \psi_{i}^{(l, P(l+1))} C_{i}(l)\right]+Q_{i}(l)+A_{i}^{\prime}(l) \psi_{i}(l, P(l+1)) A_{i}(l)$

$$
P(T)=S+\mu N^{\prime} N
$$
if

By means of Lemma in [6], we know that the equation (3.3) has a solution $K_{\theta(l)}^{(l)}$ if and only

$$
G_{\theta(l)}^{+}(l) G_{\theta(l)}(l) H_{\theta(l)}(l)=H_{\theta(l)}(l) \text { and } K_{\theta(l)}(l)=-G_{\theta(l)}^{+}(l) H_{\theta(l)}(l)+Y_{\theta(l)}(l)-G_{\theta(l)}^{+}(l) G_{\theta(l)}(l) Y_{\theta(l)}(l)
$$

Where, 


$$
\begin{aligned}
& G_{i}(l)=R_{i}(l)+B_{i}^{\prime}(l) \psi_{i}(l, P(l+1)) B_{i}(l)+D_{i}^{\prime}(l) \psi_{i}(l, P(l+1)) D_{i}(l), \\
& H_{i}(l)=B_{i}^{\prime}(l) \psi_{i}^{(l, P(l+1))} A_{i}(l)+\rho^{x u} D_{i}^{(l)} \psi_{i}(l, P(l+1)) C_{i}(l), Y_{\theta(l)}(l) \in R^{m \times n} .
\end{aligned}
$$

We substitute the above results (3.5) into (3.4) and get as follows

$$
\left\{\begin{array}{l}
P_{i}(l)=A_{i}^{\prime}(l) \psi_{i}(l, P(l+1)) A_{i}(l)+C_{i}^{\prime}(l) \psi_{i}(l, P(l+1)) C_{i}(l)+Q_{i}(l)-H_{i}^{\prime}(l) G_{i}^{+}(l) H_{i}(l) \\
H_{i}(l)=B_{i}^{\prime}(l) \psi_{i}^{(l, P(l+1))} A_{i}(l)+\rho^{x u} D_{i}^{\prime}(l) \psi_{i}(l, P(l+1)) C_{i}(l) \\
G_{i}(l)=R_{i}(l)+B_{i}^{\prime}(l) \psi_{i}(l, P(l+1)) B_{i}(l)+D_{i}^{\prime}(l) \psi_{i}(l, P(l+1)) D_{i}(l) \geq 0, l \in N_{T} \\
\mu=\frac{\operatorname{tr}\left[P_{\theta_{T}}(T)-S\right]}{\operatorname{tr}\left(N^{\prime} N\right)}
\end{array}\right.
$$

In this circumstances, we can suppose $P_{\theta(l)}(l)$ is symmetric. Otherwise, we adopt $\widetilde{P}_{\theta(l)}(l)=\frac{P_{\theta(l)}^{\prime}(l)+P_{\theta(l)}(l)}{2}$.

Now we combine the following equality

$\sum_{l=0}^{T-1} E\left[X^{\prime}(l+1) \psi_{i}(l, P(l+1)) x(l+1)-\chi^{\prime}(l) P_{i}(l) x(l)\right]=E\left[X^{\prime}(T) P_{\theta_{T}}(T) x(T)-\chi_{0} P_{\theta_{0}}{ }^{(0)} X_{0}\right]$ with (2.2) and (3.6), here $\theta(l)=i$ and we get $J\left(X_{0}, u(0), \cdots, u(T-1)\right)=\sum_{l=0}^{T-1} E\left[X^{\prime}(l) Q_{i}(l) x(l)+u^{\prime}(l) R_{i}(l) u(l)+X^{\prime}(l+1) \psi_{i}(l, P(l+1)) x(l+1)\right.$ $\left.-\chi^{\prime}(l) P_{i}(l) x(l)\right]+E\left[X^{\prime}(T)\left(S_{\theta_{T}}-P_{\theta_{T}}(T)\right) x(T)+\chi_{0} P_{\theta_{0}}{ }^{(0)} X_{0}\right]$ $=\sum_{l=0}^{T-1} E\left[X^{\prime}(l)\left(Q_{i}(l)-P_{i}(l)+A_{i}^{\prime}(l) \psi_{i}(l, P(l+1)) A_{i}(l)+C_{i}^{\prime}(l) \psi_{i}(l, P(l+1)) C_{i}(l)\right) x(l)\right.$ $+2 X^{\prime}(l)\left(A_{i}^{\prime}(l) \psi_{i}^{(l, P(l+1))} B_{i}^{(l)+} \rho^{x u} C_{i}^{\prime}(l) \psi_{i}{ }^{(l, P(l+1))} D_{i}(l)\right) u(l)+u^{\prime}(l)\left(R_{i}{ }^{(l)}+B_{i}^{\prime}(l) \psi_{i}{ }^{(l, P(l+1))} B_{i}(l)\right.$

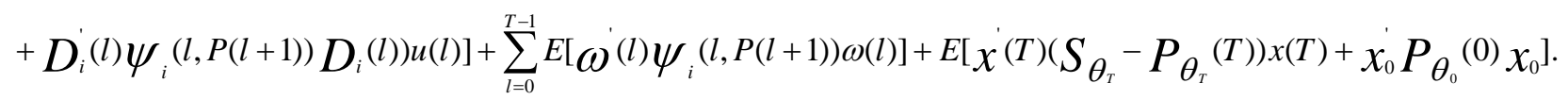
We gain as follows after the completion of square

$$
\begin{aligned}
& J\left(\chi_{0}, u(0), \cdots, u(T-1)\right)=\sum_{l=0}^{T-1} E\left[\left(u(l)+G_{i}^{+}(l) H_{i}(l) x(l)\right) G_{i}(l)\left(u(l)+G_{i}^{+}(l) H_{i}(l) x(l)\right)\right] \\
& +\sum_{l=0}^{T-1} \operatorname{tr}\left[V_{i}(l) \psi_{i}(l, P(l+1))\right]+E\left[\chi^{\prime}(T)\left(S_{\theta_{T}}-P_{\theta_{T}}(T)\right) x(T)\right]+X_{0} P_{\theta_{0}}{ }^{(0)} X_{0} .
\end{aligned}
$$

Here, $G_{i}(l), l \in N_{T}$ need to be attested. We suppose that there exists a $G_{\theta(l)}(l)$ with a negative eigenvalue $\lambda$. Let $V_{\lambda}$ become the unitary eigenvector about $\lambda$, then it purports that $V_{\lambda} V_{\lambda}=1_{\text {and }} G_{\theta(l)}(l) V_{\lambda}=\lambda V_{\lambda} \cdot$ For

$$
\begin{aligned}
& \text { any } \delta \neq 0 \text {, and ordering } \theta(l)=i \text {, let us assume that a control sequence is provided by } \\
& \tilde{u}(l)=\left\{\begin{array}{l}
-G_{i}^{+}(l) H_{i}(l) x(l), l \neq k \\
\delta|\lambda|^{-\frac{1}{2}} V_{\lambda}-G_{i}^{+}(l) H_{i}(l) x(l), l=k .
\end{array}\right.
\end{aligned}
$$

The corresponding cost as below 


$$
\begin{aligned}
& J\left(X_{0}, \tilde{u}(0), \cdots, \tilde{u}(T-1)\right)=\sum_{l=0}^{T-1} E\left[\left(u(l)+G_{i}^{+}(l) H_{i}(l) x(l)\right) G_{i}(l)\left(u(l)+G_{i}^{+}(l) H_{i}(l) x(l)\right)\right] \\
& +\sum_{l=0}^{T-1} \operatorname{tr}\left[V_{i}(l) \psi_{i}(l, P(l+1))\right]+E\left[X^{\prime}(T)\left(S_{\theta_{T}}-P_{\theta_{T}}(T)\right) x(T)\right]+X_{0} P_{\theta_{0}}{ }^{(0)} X_{0} \\
& =\left(\delta|\lambda|^{-\frac{1}{2}} V_{\lambda}\right) G_{i}(l)\left(\delta|\lambda|^{-\frac{1}{2}} V_{\lambda}\right)+\sum_{l=0}^{T-1} \operatorname{tr}\left[V_{i}(l) \psi_{i}(l, P(l+1))\right]+E\left[X^{\prime}(T)\left(S_{\theta_{T}}-P_{\theta_{T}}(T)\right) x(T)\right]+X_{0} P_{\theta_{0}}{ }^{(0)} X_{0} \\
& =-\delta^{2}+\sum_{l=0}^{T-1} \operatorname{tr}\left[V_{i}(l) \psi_{i}(l, P(l+1))\right]+E\left[X^{\prime}(T)\left(S_{\theta_{T}}-P_{\theta_{T}}(T)\right) x(T)\right]+X_{0} P_{\theta_{0}}{ }^{(0)} X_{0} .
\end{aligned}
$$

When $\delta \rightarrow \infty$,it makes $J\left(x_{0}, \tilde{u}(0), \cdots, \tilde{u}(T-1)\right) \rightarrow-\infty$, which is in contradiction with the attainability on the linear quadratic problem (2.1)-(2.3).

Through the above interpretation and (3.4), we can get the optimal value $V_{i}\left(X_{0}\right)$ as follows

$$
V_{i}\left(X_{0}\right)=J\left(X_{0}, U_{*}(0), \cdots, U_{*}(T-1)\right)=\sum_{l=0}^{T-1} \operatorname{tr}\left[V_{i}(l) \psi_{i}(l, P(l+1))\right]+X_{0} P_{\theta_{0}}{ }^{(0)} X_{0}-\mu t r(M) .
$$

The process of proof is complete.

In the ending, we will use the following result, which offers an equivalent relation between the solvability of the GDRE and the well-posedness of the linear quadratic problem.

Theorem 3.2 The linear quadratic problem (2.1)-(2.3) is well-posed, hence there are solutions $\left(P_{\theta(l)}(l), \mu\right)$

on the GDRE (3.1). On the contrary, if the GDRE (3.1) has solutions $\left(P_{\theta(l)}(l), \mu\right)$, then the linear quadratic

problem (2.1)-(2.3) is well-posed. Furthermore, the optimal cost satisfies

$V_{i}\left(X_{0}\right)=\sum_{l=0}^{T-1} \operatorname{tr}\left[V_{i}(l) \psi_{i}(l, P(l+1))\right]+X_{0} P_{\theta_{0}}{ }^{(0)} X_{0}-\mu t r(M)$.

Proof Necessity part. Think about the following cost from $k$ to $T$

$W_{\theta(k)}^{k}(x(k))=\inf _{u(0), \cdots, u(T-1)} \sum_{l=k}^{T-1} E\left[x^{\prime}(l) Q_{\theta(l)} x(l)+u^{\prime}(l) R_{\theta(l)} u(l)+x^{\prime}(T) S_{\theta_{T}} x(T)\right]$

In the light of the optimal standard, if $W_{\theta(k)}^{k}(x(k))$ is finite, and there is $W_{\theta(m)}^{m}(x(m))$ for any $m \geq k$.

Owing to $W_{\theta_{0}}^{0}(x(0))$ is finite, we could deduce that $W_{\theta(k)}^{k}(x(k))$ is finite for any $T-1 \geq k \geq 0$.

Let $k=T-1_{\text {and }} P_{\theta_{T}}(T)=S_{\theta_{T}}+\mu N^{\prime}{ }^{\prime}$. By means of (2.1) and (3.7), we get as follows

$$
W_{\theta(T-1)}^{T-1}(x(T-1))-\operatorname{tr}\left[V_{\theta(T-1)}(T-1) P_{\theta_{T}}(T)\right]-E\left[\chi^{\prime}(T)\left(S_{\theta_{T}}-P_{\theta_{T}}(T)\right) x(T)\right]
$$$$
=\inf _{u(T-1)} E\left[X ^ { \prime } ( T - 1 ) \left(Q_{\theta(T-1)}(T-1)+A_{\theta(T-1)}^{\prime}(T-1) P_{\theta_{T}}(T) A_{\theta(T-1)}(T-1)\right.\right.
$$$$
\left.+C_{\theta(T-1)}^{\prime}(T-1) P_{\theta_{T}}(T) C_{\theta(T-1)}(T-1)\right) x(T-1)+2 X^{\prime}(T-1)\left(A_{\theta(T-1)}^{\prime}(T-1) P_{\theta_{T}}(T) B_{\theta(T-1)}(T-1)\right.
$$$$
\left.+\rho^{x u}(T-1) C_{\theta(T-1)}^{\prime}(T-1) P_{\theta_{T}}(T) D_{\theta(T-1)}(T-1)\right) u(T-1)+u^{\prime}(T-1)\left(R_{\theta(T-1)}(T-1)\right.
$$$$
\left.\left.+B_{\theta(T-1)}^{\prime}(T-1) P_{\theta_{T}}(T) B_{\theta(T-1)}(T-1)+D_{\theta(T-1)}^{\prime}(T-1) P_{\theta_{T}}(T) D_{\theta(T-1)}(T-1)\right) u(T-1)\right] \text {. }
$$

Following Lemma in [6] to the above quadratic equation, we obtain a symmetric matrix $P_{\theta(T-1)}(T-1)$ as below 
$W_{\theta(T-1)}^{T-1}(x(T-1))-\operatorname{tr}\left[V_{\theta(T-1)}(T-1) P_{\theta_{\tau}}(T)\right]-E\left[X^{\prime}(T)\left(S_{\theta_{\tau}}-P_{\theta_{\tau}}(T)\right) x(T)\right]=E\left[X^{\prime}(T-1) P_{\theta(T-1)}(T-1) x(T-1)\right]$

$P_{\theta(T-1)}(T-1)=Q_{\theta(T-1)}(T-1)+A_{\theta(T-1)}^{\prime}(T-1) P_{\theta_{T}}(T) A_{\theta(T-1)}(T-1)+C_{\theta(T-1)}^{\prime}(T-1) P_{\theta_{T}}(T) C_{\theta(T-1)}(T-1)$

$-H_{\theta(T-1)}^{\prime}(T-1) G_{\theta(T-1)}^{+}(T-1) H_{\theta(T-1)}(T-1)$

$G_{\theta(T-1)}(T-1)=R_{\theta(T-1)}(T-1)+B_{\theta(T-1)}^{\prime}(T-1) P_{\theta_{\tau}}(T) B_{\theta(T-1)}(T-1)+D_{\theta(T-1)}^{\prime}(T-1) P_{\theta_{r}}(T) D_{\theta(T-1)}(T-1) \geq 0$

$H_{\theta(T-1)}(T-1)=B_{\theta(T-1)}^{\prime}(T-1) P_{\theta_{T}}(T) A_{\theta(T-1)}(T-1)+\rho^{x u} D_{\theta(T-1)}(T-1) P_{\theta_{T}}(T) C_{\theta(T-1)}(T-1)$.

Obviously, the above form is GDRE (3.1) for $l=T-1$.

Fixing $\theta(l)=i$, suppose that GDRE (3.1) possesses a set of solutions $\left(P_{\theta(l)}(l), \mu\right)$ with $l=k, \cdots, T-1$.

$W_{i}^{k}(x(k))-\operatorname{tr}\left[V_{i}(k) \psi_{i}(k, P(k+1))\right]-E\left[X^{\prime}(T)\left(S_{\theta_{T}}-P_{\theta_{T}}(T)\right) x(T)\right]=E\left[X^{\prime}(k) P_{i}(k) x(k)\right]$.

By means of (3.7), we get

$W_{i}^{k-1}(x(k-1))-\sum_{l=k-1}^{T-1} \operatorname{tr}\left[V_{i}(l) \psi_{i}(l, P(l+1))\right]-E\left[X^{\prime}(T)\left(S_{\theta_{T}}-P_{\theta_{T}}(T)\right) x(T)\right]$

$=\inf _{u(k-1)} E\left[X^{\prime}(k-1)\left(Q_{\theta(k-1)}(k-1)+\dot{A_{\theta(k-1)}^{\prime}}(k-1) P_{\theta(k-1)}(k-1) A_{\theta(k-1)}(k-1)\right.\right.$

$\left.+C_{\theta(k-1)}^{\prime}(k-1) P_{\theta(k-1)}(k-1) C_{\theta(k-1)}(k-1)\right) x(k-1)+2 X^{\prime}(k-1)\left(A_{\theta(k-1)}^{\prime}(k-1) P_{\theta(k)}(k) B_{\theta(k-1)}(k-1)\right.$

$\left.+\rho^{x u} C_{\theta(k-1)}^{\prime}(k-1) P_{\theta(k)}(k) D_{\theta(k-1)}(k-1)\right) u(k-1)+u^{\prime}(k-1)\left(R_{\theta(k-1)}(k-1)\right.$

$\left.\left.+B_{\theta(k-1)}^{\prime}(k-1) P_{\theta(k)}(k) B_{\theta(k-1)}(k-1)+D_{\theta(k-1)}^{\prime}(k-1) P_{\theta(k)}(k) D_{\theta(k-1)}(k-1)\right) u(k-1)\right]$.

From Lemma in [6], it is evident that the finiteness of $V_{\theta(T-1)}^{k-1}(k-1)$ is equivalent to the following

$$
\left\{\begin{array}{l}
P_{\theta(k-1)}(k-1)=A_{\theta(k-1)}^{\prime}(k-1) P_{\theta(k)}(k) A_{\theta(k-1)}(k-1)+C_{\theta(k-1)}^{\prime}(k-1) P_{\theta(k)}(k) C_{\theta(k-1)}(k-1) \\
+Q_{\theta(k-1)}(k-1)-H_{\theta(k-1)}^{\prime}(k-1) G_{\theta(k-1)}^{+}(k-1) H_{\theta(k-1)}(k-1) \\
H_{\theta(k-1)}(k-1)=B_{\theta(k-1)}^{\prime}(k-1) P_{\theta(k)}(k) A_{\theta(k-1)}(k-1)+\rho^{x u} D_{\theta(k-1)}(k-1) P_{\theta(k)}(k) C_{\theta(k-1)}(k-1) \\
G_{\theta(k-1)}(k-1)=R \theta(k-1) \\
G_{\theta(k-1)}(k-1) G_{\theta(k-1)}^{+}(k-1) H_{\theta(k-1)}(k-1)-H_{\theta(k-1)}^{\prime}(k-1) P_{\theta(k)}(k) B_{\theta(k-1)}(k-1)+D_{\theta(k-1)}^{\prime}(k-1) P_{\theta(k)}(k) D_{\theta(k-1)}(k-1)
\end{array}\right.
$$

Furthermore,

$W_{i}^{k-1}(x(k-1))=\sum_{l=k-1}^{T-1} \operatorname{tr}\left[V_{i}(l) \psi_{i}(l, P(l+1))\right]+E\left[X^{\prime}(T)\left(S_{\theta_{T}}-P_{\theta_{T}}(T)\right) x(T)\right]+\chi^{\prime}(k) P_{i}(k) x(k)$.

Sufficiency part. Let

$L_{i}(l, P)=A_{i}^{\prime}(l) \psi_{i}(l, P(l+1)) A_{i}(l)-P_{i}(l)+C_{i}^{\prime}(l) \psi_{i}(l, P(l+1)) C_{i}(l)-Q_{i}(l)$

$M_{i}(l, P)=A_{i}^{\prime}(l) \psi_{i}(l, P(l+1)) B_{i}(l)+\rho^{x u} C_{i}^{\prime}(l) \psi_{i}(l, P(l+1)) D_{i}(l)$

$N_{i}(l, P)=R_{i}(l)+B_{i}^{\prime}(l) \psi_{i}{ }^{(l, P(l+1))} B_{i}(l)+D_{i}^{\prime}(l) \psi_{i}(l, P(l+1)) D_{i}(l)$.

We suppose $P_{\theta_{1}}(1), \cdots, P_{\theta_{T}}{ }^{(T)}$ satisfy

$\left[\begin{array}{cc}L_{i}(l, P) & M_{i}(l, P) \\ M_{i}^{\prime}(l, P) & N_{i}(l, P)\end{array}\right] \geq 0$

for $l=0,1, \cdots, T-1$ and $P_{\theta_{T}}(T) \leq S+\mu N^{\prime} N$.

We have known the following 


$$
\begin{aligned}
& J\left(X_{0}, u(0), \cdots, u(T-1)\right)=\sum_{l=0}^{T-1} E\left[\left(\chi^{\prime}(l) Q_{i}(l) x(l)+u^{\prime}(l) R_{i}(l) u(l)\right)+\chi^{\prime}(T) S_{\theta_{T}}{ }^{x(T)}\right] \\
& =\sum_{l=0}^{T-1} E\left[\chi^{\prime}(l)\left(Q_{i}(l)-P_{i}(l)+A_{i}^{\prime}(l) \psi_{i}(l, P(l+1)) A_{i}(l)+C_{i}^{\prime}(l) \psi_{i}(l, P(l+1)) C_{i}(l)\right) x(l)\right. \\
& +2 \chi^{\prime}(l)\left(A_{i}^{\prime}(l) \psi_{i}(l, P(l+1)) B_{i}(l)+\rho^{x u} C_{i}^{\prime}(l) \psi_{i}(l, P(l+1)) D_{i}(l)\right) u(l)+u^{\prime}(l)\left(R_{i}(l)+B_{i}^{\prime}(l) \psi_{i}(l, P(l+1)) B_{i}(l)\right. \\
& \left.\left.+D_{i}^{\prime}(l) \psi_{i}(l, P(l+1)) D_{i}(l)\right) u(l)\right]+\sum_{l=0}^{T-1} E\left[V_{i}(l) \psi_{i}(l, P(l+1))\right]+E\left[\chi^{\prime}(T)\left(S_{\theta_{T}}-P_{\theta_{T}}(T)\right) x(T)+\chi_{0} P_{\theta_{0}}{ }^{(0)} \chi_{0}\right] .
\end{aligned}
$$

We can reach the following from Extended Schur's Lemma in [5].

$$
J\left(X_{0}, u(0), \cdots, u(T-1)\right) \geq \sum_{l=0}^{T-1} \operatorname{tr}\left[V_{i}(l) \psi_{i}(l, P(l+1))\right]+\chi_{0} P_{\theta_{0}}{ }^{(0)} X_{0}-\mu \operatorname{tr}(M) .
$$

That is to say,

$$
V_{i}\left(X_{0}\right)=\sum_{l=0}^{T-1} \operatorname{tr}\left[V_{i}(l) \psi_{i}(l, P(l+1))\right]+\chi_{0} P_{\theta_{0}}(0) X_{0}-\mu t r(M)
$$

which indicates that the linear quadratic problem (2.1) - (2.3) is well-posed.

The next content will be the main result about this section.

Theorem 3.3 The nether conclusions are equivalent:

(i) The linear quadratic problem (2.1) - (2.3) is attainable.

(ii) The linear quadratic problem (2.1) - (2.3) is well-posed.

(iii) The GDRE (3.1) is solvable.

Moreover, the feedback control law is accomplished by

$$
u(l)=-\left[R_{i}(l)+B_{i}^{\prime}(l) \psi_{i}(l, P(l+1)) B_{i}(l)+D_{i}^{\prime}(l) \psi_{i}(l, P(l+1)) D_{i}(l)\right]^{+}
$$

$\left[B_{i}^{\prime}(l) \psi_{i}(l, P(l+1)) A_{i}(l)+\rho^{x u} D_{i}(l) \psi_{i}(l, P(l+1)) C_{i}(l)\right] x(l)$,

here, $P_{\theta_{0}}(0), \cdots, P_{\theta_{T}}(T)$ are solutions on the GDRE (3.1), at the same time, $l \in N_{T}$.

Proof By Theorem 3.2, we can educe that (ii) is equivalent to (iii). Next our assignment is to testify that (i) is equivalent to (iii). However, from Theorem 3.1, we only need to attest (iii) $\Rightarrow$ (i).

Assume the GDRE (3.1) has a series of solutions $\left(P_{\theta_{1}}(1), \cdots, P_{\theta_{T}}(T), \mu\right)$. We can prove the following

equation on the basis of the Theorem 3.1

$J\left(\chi_{0}, u(0), \cdots, u(T-1)\right)=\sum_{l=0}^{T-1} E\left[X^{\prime}(l) Q_{i}(l) x(l)+u^{\prime}(l) R_{i}(l) u(l)+\chi^{\prime}(T) S_{\theta_{T}}{ }^{(T)}\right]$

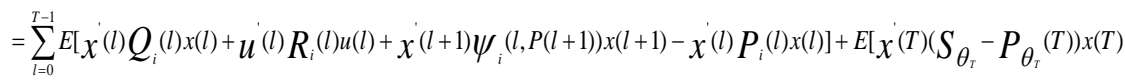

$\left.+{ }^{\prime} P_{0} P_{\theta_{0}}(0) X_{0}\right]=\sum_{l=0}^{T-1} E\left[\left(u(l)+G_{i}^{+}(l) H_{i}(l) x(l)\right) G_{i}(l)\left(u(l)+G_{i}^{+}(l) H_{i}(l) x(l)\right)\right]$

$+\sum_{l=0}^{T-1} \operatorname{tr}\left[V_{i}(l) \psi_{i}(l, P(l+1))\right]+E\left[X^{\prime}(T)\left(S_{\theta_{\tau}}-P_{\theta_{r}}(T)\right) x(T)\right]+X_{0} P_{\theta_{0}}{ }^{(0)} X_{0}$.

Hence, the optimal value

$V_{i}\left(X_{0}\right)=\sum_{l=0}^{T-1} \operatorname{tr}\left[V_{i}(l) \psi_{i}(l, P(l+1))\right]+X_{0} P_{\theta_{0}}{ }^{(0)} X_{0}-\mu \operatorname{tr}(M)$,

and the feedback $u(l)=-G_{i}^{+}(l) H_{i}(l) x(l)$.

\section{References}

[1] H. Dong, Z. Wang, D.W.C. Ho and H. Gao. Variance-constrained $H_{\infty}$ filtering for a class of nonlinear time-varying systems with multiple missing measurements: the finite-horizon case. IEEE Transactions on Signal Processing. Vol.58(5)(2010), 2534-2543. 
[2] L. Ma, Y. Bo, Y. Zhouand Z. Guo. Error variance-constrained $H_{\infty}$ filteringfor a class of nonline ar stochastic systems with degraded measurements: the finite-horizoncase. International Journal of Systems Science. Vol.43(12)(2012), 2361-2372.

[3] D.G. Luenberger. Optimization by vector space methods. New York: Wiley, (1968).

[4] R.Penrose. A generalized inverse of matrices. Proc. Cambridge Philos. Soc. Vol.52(1955), 17-19.

[5] A. Albert. Conditions for positive and nonnegative definitesness in terms of pseudo-inverse. SIAM J. Appl. Math. Vol.17(1969), 434-440.

[6] M.A. Rami, X. Chen and X.Y. Zhou. Discrete-time indefinite LQ control with state and control dependentnoises. J.Glob.Optim. Vol.23(2002), 245-265. 